\title{
EL STORYTELLING EN LA MARCA DE DESTINOS TURÍSTICOS: EL CASO DE GIRONA
}

\author{
Raquel Camprubi* \\ Universitat de Girona \\ https://orcid.org/0000-0001-7084-7889
}

Cèlia Planas

Universitat de Girona

\section{RESUMEN}

El storytelling como estrategia para transmitir los valores e identidad de una marca ha adquirido importancia en los últimos años, pero aún hay poca literatura que desarrolle este concepto entorno a los destinos turísticos. Este artículo pretende analizar las potencialidades y uso del storytelling en un destino turístico a través de relatos y leyendas tradicionales. Las leyendas de la ciudad de Girona (España) se utilizaron como caso de estudio. Los resultados ponen en evidencia que, a pesar del potencial de las leyendas, actualmente no se está explotando adecuadamente.

Palabras clave: storytelling; leyendas; branding; marca; destino turístico.

\section{Storytelling in tourism destinations branding: the case of Girona}

\section{ABSTRACT}

Storytelling as strategy to transmit values and identity of a brand has acquired relevance during the last years. However, this concept is already relatively underdeveloped in academic literature on tourism destinations. This paper aims to analyze storytelling potentialities and use in a tourism destination trough traditional stories. Legends of the city of Girona (Spain) have been chosen as a case study. Results demonstrated that legends of the city have the potential to develop a storytelling strategy, but this potential is not used properly.

Keywords: storytelling; legends; branding; brand; tourism destination.

Fecha de recepción: 3 de mayo de 2019

Fecha de aceptación: 31 de octubre de 2019

* Facultad de Turismo. Universitat de Girona. Plaça Ferrater Mora, 1. 17004 GIRONA (España).

E-mail: raquel.camprubi@udg.edu,celiaplanas.ar@gmail.com 


\section{INTRODUCCIÓN}

Es bien conocida la particularidad del producto turístico en el que su compra se basa en las expectativas del turista, es decir, de la imagen preconcebida del destino en su mente (Kotler, Haider y Rein, 1993). A este hecho cabe añadir que, el turista, ya no adquieren solamente un producto sino que lo compra pensando en adquirir la experiencia que se encuentra detrás de cada producto turístico, y que pretende disfrutar durante sus vacaciones en ese destino (Woodside y Megehee, 2010). Eso requiere que las organizaciones que se encargan de gestionar los diferentes destinos turísticos deben ser capaces de crear una potente imagen de marca que permita atraer la atención del turista e influenciarle en el proceso de decisión y de compra de sus vacaciones (Gartner, 1994; Pritchard y Morgan, 2004).

Por consiguiente, una estrategia de branding bien estructurada y ejecutada con éxito contribuye favorablemente al valor, la reputación y el poder competitivo de cualquier destino (Yavuz et al., 2016). Sin embargo, el nombre del destino ya no es suficiente para diferenciarlo y asociar determinadas características para conseguir el posicionamiento deseado, así como otros aspectos que contribuyen a la complejidad del branding en un territorio (Pike, 2005). Es en este contexto que los éxitos proporcionados por el storytelling en las marcas de productos tangibles (Salomon, 2007) han brindado una nueva oportunidad para el uso de esta herramienta en los destinos turísticos de todo el mundo, siendo este un fenómeno relativamente reciente (Mossberg, Therkelsen, Huijbens, Björk y Olson, 2011; Keskin, Akgun, Zehir y Ayar, 2016; Yavuz, Sumbul, Elpeze, y Derdiyok, 2016). El storytelling es una técnica que permite a las organizaciones gestoras de destinos turísticos comunicar, de forma efectiva, su marca turística a través de la conversión de historias y mitos en experiencias tangibles (Yavuz et al., 2016) que junto con su correspondiente argumento y varios personajes permiten al turista conocer mejor el destino al que viajan. El storytelling posibilita que, una marca o destino turístico sea diferente al resto de competidores y gane un valor añadido positivo (Yavuz et al., 2016) a través del aprovechamiento de unos recursos intangibles, como pueden ser los mitos y las leyendas propios de cada destino turístico y pertenecientes a su cultura y sus tradiciones particulares. Además, "la importancia del storytelling como recursos reside en parte en la creencia que sus cualidades particulares contribuyen a realzar el valor de la experiencia turística" (Mathisen, 2014: 157).

Akgün et al. (2015) y Mossberg et al. (2011) afirman que, en los últimos años el storytelling ha ganado cada vez más atención como una tendencia de marketing global en la industria del turismo; sin embargo, aún no existe una considerable indagación sobre el storytelling en relación directa con el branding de destinos turísticos en la literatura académica (Yavuz et al., 2016; Keskin, et al., 2016).

En este contexto, el presente artículo nace del interés por conocer como un destino turístico puede aplicar el storytelling como estrategia de marketing, utilizando historias y leyendas que reflejen la historia cultural del destino. Con este propósito se ha utilizado el caso de estudio de las leyendas de la ciudad de Girona (Cataluña, España), en el que se ha analizado una muestra de leyendas tradicionales de la ciudad para comprobar su posible 
uso y aplicación a la hora de comunicar la marca del destino, y se ha contrastado con la actual estrategia de marca mediante entrevistas en profundidad.

El presente artículo se estructura de la siguiente manera. En primer lugar, se realiza una revisión de la literatura, poniendo énfasis en los conceptos de la marca e identidad de marca y la marca territorial. Seguidamente, el discurso se centra en poner de relieve el storytelling y su desarrollo como estrategia en el contexto de las marcas de destinos turísticos. En segundo lugar, se describe la metodología seguida, para pasar a la presentación de los resultados en el cuarto apartado. Finalmente, se presentan las conclusiones basadas en el análisis del caso y algunas vías para la investigación futura en este campo.

\section{EL STORYTELLING}

\subsection{La marca y la identidad de marca}

Según Velilla (2010), el concepto de marca (del inglés brand) se relaciona con la palabra escandinava "brandr", que significa quemar, o con las alemanas "es brennte" (se está quemando) o "der Brand (el fuego) y se puede asociar con el uso del fuego para dejar una marca distintiva en un objeto o con la tradición de usar el fuego para indicar la propiedad del ganado (Velilla, 2010).

El concepto de marca no se empezó a utilizar en marketing hasta el siglo XX y, a parte de denominar objetos también les agregaba un valor añadido, haciendo que, cada vez más los clientes se guiaran por las marcas en sus compras y no por los métodos de venta (Bastos y Levy, 2012). Las marcas han sido entendidas como un símbolo que identifica y deferencia a los productos y a sus competidores (Velilla, 2010; Aaker 1998). No obstante, hiendo más allá, en la actualidad se considera que las marcas se definen no solo por la descripción de un producto, sino por el conjunto de ideas que permanecen en la mente de su público; teniendo en cuenta que la marca no solo está formada por su propia descripción, sino también por como sus consumidores la perciben (Velilla, 2010). Según Fennell (1978), a raíz de un problema surge una necesidad que impulsa la motivación para crear una marca (citado en Park, Jaworski, y MacInnis, 1986) junto con un logotipo, un nombre, un empaquetado, un diseño y una estrategia de comunicación. Estos elementos o signos son consecuencia de una estrategia de branding (Chaves, 2017) que bien definida permitirá construir una ventaja distintiva y sostenible (Rooney, 1995) Así, pues, para establecer una marca hace falta definir unos valores y una personalidad que transmitan a su audiencia aquello que la misma marca representa y quiere difundir (Kotler, 2011; Bastos y Levy, 2012) y para ello es necesario que se diferencie de su competencia y que se cree un vínculo emocional entre la marca y su público y, que a su vez actúe como razón de preferencia a la hora de escoger entre dos marcas (Chaves, 2017).

Los signos mencionados anteriormente son los que ayudan a plasmar la identidad corporativa de una marca; junto con otras piezas tangibles e intangibles, como podrían ser los trabajadores o los valores de la organización (Balmer, 2001). Las marcas parten de una identidad que es considerada una esencia atemporal, que no se modifica a lo largo de los años, los elementos clave son los objetivos y valores de esta (Urde, 2013), es decir, los valores y características que definen una marca son los elementos que se 
quieren transmitir al consumidor (Blombäck y Ramírez-Pasillas, 2012). En consecuencia, cuando la identidad de la marca es comunicada de manera efectiva se crea un hito en la mente de los consumidores (Urde, 2013), que contribuye a la creación de una construcción social de la misma (Berger y Luckman, 1991). Aún así, hay que considerar que las identidades de marca no se pueden entender como un elemento estático, ya que pueden ser interpretadas de diferentes maneras según la audiencia (Blombäck y Ramírez-Pasillas, 2012), o bien, evolucionar a lo largo del tiempo. En cualquier caso, una vez definida la identidad corporativa de la marca de forma efectiva, esto permite a los consumidores establecer un vínculo emocional con la marca y posicionarla en relación a otras marcas competidoras (Balmer, 2010).

\subsection{La marca de territorio}

Los territorios, entendidos como espacios geográficos de diversa índole tales como países, regiones o ciudades, se han convertido en objetos de consumo (Urry, 1995), para el deleite de turistas y visitantes que ansían poder llenarse de experiencias positivas en los lugares visitados. A su vez, desde los territorios se ve esta llegada de turistas y visitantes como un potencial de desarrollo y beneficios económicos para sus residentes. No obstante, los territorios son cada vez más sustituibles y difíciles de diferenciar (Pike, 2005). Es en este contex to que la construcción y proyección de una marca fuerte para el territorio puede contribuir positivamente a incrementar su competitividad (Miličević, Mihalič, y Sever, 2017), dadas las ventajas referidas anteriormente.

El branding asociado a lugares (place branding) se puede definir como el proceso de construir una marca para un lugar basado en la identidad del territorio i en la formación de una imagen positiva en la mente de los stakeholders (Anholt, 2010). La singularidad y complejidad del proceso de creación de marca en un territorio es evidente y distinta al proceso que se produce en un contexto corporativo, aunque los objetivos de ambas aproximaciones tengan un objetivo terminal común (De San Eugenio, 2013). En este contexto, Govers y Go (2004) expresan la relativa sencillez para una empresa en crear una marca que represente su personalidad para hacerla más humana. Estos autores, por el contrario, remarcan que los lugares son humanos desde su inicio, con personalidades heterogéneas que se han ido forjando a lo largo de la historia, y pueden producir confusión. A este hecho cabe añadir otros elementos que aportan complejidad al branding de territorios como la naturaleza del dominio público en los lugares, la heterogeneidad de públicos internos (residentes y empresas locales) y externos (turistas y visitantes) (San Eugenio, 2013), la multi-dimensionalidad del producto turístico (Pike, 2005) y su inherente intangibilidad (De San Eugenio, 2013), así como la existencia de múltiples agentes que interaccionan en el territorio simultáneamente (Camprubí et al., 2008).

Para el desarrollo de la identidad de la marca del territorio, tal y como se desprende de lo mencionado anteriormente, también existe una mayor complejidad. Los territorios no pueden desarrollar una identidad nueva o ficticia como en el caso de las empresas, sino que deben tomar como base la identidad del lugar. Se trata pues de una identidad colectiva o social que representa a los distintos stakeholders del destino (Meler y Ruzic, 1999; van Rekom y Go, 2006; Ruiz y Hernández, 2007) y se ha construido a través de 
los discursos históricos, políticos, religiosos y culturales (Govers, Go, 2016) a lo largo del tiempo, de modo que la identidad del territorio se postula como un proceso interactivo (Kavaratzis y Hatch, 2013). En este sentido, Kavaratzis y Hatch (2013: 74) destacan que el branding territorial se centra en comunicar la marca del territorio a través de los medios de comunicación y los mensajes que se consideran más adecuados para articular y reforzar la identidad del lugar, y tienen el poder para convencer al público objetivo.

\subsection{El storytelling}

El storytelling se erige como parte de la estrategia del marketing de contenidos, y se define como "la forma de compartir y combinar conocimientos y experiencias a través de narraciones y anécdotas para comunicar ideas complejas, conceptos y conexiones causales y construir conexiones y asociaciones" (Keskin et al., 2016: 32), es decir, se trata del arte de fabricar historias con el propósito de influir a las audiencias (Salomon, 2007).

En su forma original el storytelling se identifica alrededor de las hogueras, mientras las mujeres cocinaban y los hombres de las tribus contaban leyendas e historias de sus antepasados. A través de esas historias se transferían los conocimientos de generación en generación, formaban la identidad de la tribu y, a su vez, les proporcionaban unos valores que podía diferenciarlos de las tribus rivales (Fog, Budtz, Munch, y Blanchette, 2010). En la vida moderna, el storytelling se ha convertido en una pieza clave para el mundo empresarial, a consecuencia de la necesidad de diferenciarse de sus competidores, y ofrecer un valor añadido en la experiencia de sus clientes (Frog, Budtz y Yakaboylu, 2005). El storytelling difunde historias sobre una empresa ayudándola a crear y fomentar sus valores, su cultura, y su identidad, es decir, contribuye a generar un relato de la marca empresarial y/o de sus productos, que le permite atraer la atención de los consumidores y favorece su fidelización (Pulizzi, 2012). Es por este motivo que el storytelling se considera una herramienta estratégica con un gran potencial dentro del proceso de creación de la marca de una empresa o de sus productos (Akgun et al., 2016; Fog et al., 2010). Así pues, el mundo empresarial ha encontrado en el storytelling una herramienta para realzar la marca como ventaja competitiva y, por ende, mejorar su posicionamiento en el mercado.

En este contexto, ha nacido el llamado "storytelling management", el cual pretende dar valor y orientar la creación del relato (Salomon, 2007). Para la construcción del relato, Fog et al. (2010) apunta que el storytelling se compone de cuatro elementos clave: el mensaje, el conflicto, los personajes y el argumento; coincidiendo con Yavuz et al. (2016) que también determinan que todas las historias contienen un tema, personajes, mensajes y conflictos/experiencias, los cuales pueden ser positivos, negativos o emocionales. Para que el relato sea efectivo, Pinzaru (2012) remarca la importancia de los sentimientos y emociones en las narraciones, así como el hecho que deben contextualizarse desde la perspectiva de la experiencia personal, ya que son más creíbles y permiten que el consumidor se pueda sentir identificado; sobre todo teniendo en cuenta que el consumidor ya no compra un producto o una marca, sino las historias que representan, sus mitos y sus arquetipos (Ramzy, 2006). Así pues, las campañas publicitarias se han convertido en secuencias narrativas (Salomon, 2007), que poco a poco construyen el valor de la marca, con la propia identificación de sus consumidores, 
proporcionando la construcción de una relación favorable entre la marca y el consumidor (Woodside et al. 2008).

\subsection{El storytelling en turismo}

Los turistas reciben una gran cantidad de información sobre los diferentes destinos que, muchas veces comparten características similares. Por este motivo, cada uno de los destinos pretende ser diferente del resto para conseguir ser la elección final del turista a la hora de viajar.

Según Morrisson y Anderson (2002), la marca de un destino turístico se puede definir como la manera en la que el mismo destino comunica su identidad para diferenciarse del resto. Esto hace imprescindible la función de identificarse y diferenciarse del resto de destinos (Qu, Kim, y Im, 2011). Cada vez más, se puede observar la necesidad de distribuir experiencias y, para esa distribución, el storytelling ha sido una de las herramientas que se ha utilizado de forma efectiva y, además, que está logrando más relevancia actualmente (Chronis, 2008). Según Chronis (2008), un destino turístico puede actuar como un escaparate capaz de mostrar a sus visitantes su entorno físico a través de historias y también difundir su historia, cultura o tradiciones. En esta línea, Kim y Youn (2017) concluyen que una historia real o imaginaria sobre un destino turístico puede transformarse en una ventaja competitiva y, por tanto, convertirse en una virtud diferenciadora de otros destinos rivales. Según Kim y Youn (2017), hacen falta tres factores para que una historia pueda suponer una ventaja competitiva para un destino: autenticidad, emociones e información. Es decir, si una marca consigue crear una historia capaz de ser percibida como autentica por sus consumidores, conseguirá que estos se sientan integrados y conectados con la historia de la marca (Guber, 2007) e involucrados en el destino (Kim y Youn, 2017). Por tanto, el relato debe contemplar la estética percibida, la estructura narrativa y las autoreferencias como componentes clave en el storytelling turístico (Akgün et al., 2015). De modo que cuantos más detalles incluya la historia (nombres, años, lugares, etc.,) más creíble y autentica resultará (Chiu, Hsieh y Kuo, 2012), y a su vez, cuando genere algún tipo de emoción será más fácil de recordar (Akgün et al., 2015; Kensinger and Corkin, 2003).

Aunque el estudio del storytelling ya cuenta con una dilatada trayectoria en el contexto empresarial y organizativo, se encuentra aún pocas investigaciones que pongan énfasis en los valores que puede aportar en el desarrollo del branding de un destino turístico (Mossberg, et al., 2011; Keskin et al., 2016; Yavuz et al., 2016). Así pues, este estudio se postula como una contribución a la literatura académica en este campo.

\section{METODOLOGÍA}

La ciudad de Girona, ubicada en el nordeste de Cataluña (España), se ha tomado como caso de estudio, con el fin de poder evaluar en qué medida las leyendas tradicionales de la ciudad se han incorporado en el storytelling de la marca de la ciudad. Las Leyendas de Girona son relatos que se han ido transmitiendo a lo largo de los años y que se han utilizado tanto en la promoción como en la divulgación de cultura y las tradiciones de la ciudad de Girona. En total se han tomado como muestra de referencia 21 leyendas de 
las 34 leyendas existentes. La determinación de la muestra se ha basado en la revisión de la web oficial de turismo del Ayuntamiento de Girona y de la Asociación de Guias de Girona, así como de los libros "Llegendes de Girona. Itinerari pel Barri Vell" (García, 1956) y "Llegendes i misteris de Girona" (Vivó, 1989). Se han escogido las 21 leyendas con una mayor recurrencia en las distintas fuentes consultadas, o bien aquellas que tenían una estrecha vinculación con elementos patrimoniales de la ciudad (cuadro 1).

\section{Cuadro 1}

\section{MUESTRA DE LAS LEYENDAS DE GIRONA}

\begin{tabular}{|l|l|}
\hline Título de la leyenda & Referencias dónde se menciona \\
\hline El toro de oro & $\begin{array}{l}\text { Ajuntament de Girona (2018a); Garcia (1956); Vivó } \\
(1989)\end{array}$ \\
\hline El jefe del ayuntamiento & Garcia (1956) \\
\hline La calle del lobo & Garcia (1956); Vivó (1989) \\
\hline El Halcón del "Cap d'Estopes” & $\begin{array}{l}\text { Ajuntament de Girona (2018a); Ajuntament de } \\
\text { Girona,(2018b); Associació Guies de Girona (2018); } \\
\text { Garcia (1956); Vivó (1989) }\end{array}$ \\
\hline El fantasma de la judía & Vivó (1989) \\
\hline El Tarlà de la Platería & $\begin{array}{l}\text { Ajuntament de Girona (2018a); Ajuntament de } \\
\text { Girona (2018b); Associació Guies de Girona (2018); } \\
\text { Garcia (1956); Vivó (1989) }\end{array}$ \\
\hline El vampiro de la Rambla & Ajuntament de Girona (2018b); Garcia (1956) \\
\hline El “Banyeta” & $\begin{array}{l}\text { Ajuntament de Girona (2018b); Associació Guies de } \\
\text { Girona (2018); Garcia (1956) }\end{array}$ \\
\hline Gerió, fundador de Girona & Ajuntament de Girona (2018a); Vivó (1989) \\
\hline El emperador de la barba florecida & Vivó (1989) \\
\hline La espada de Carlomagno & Garcia (1956) \\
\hline La bruja de la Catedral & $\begin{array}{l}\text { Ajuntament de Girona (2018a); Ajuntament de } \\
\text { Girona (2018b); Associació Guies de Girona (2018); } \\
\text { Garcia, 1956; Vivó (1989) }\end{array}$ \\
\hline La silla de Carlomagno & Garcia (1956); Vivó (1989) \\
\hline La campana Benita (Ángeles y campanas) & Garcia (1956); Vivó (1989) \\
\hline Ajuntament de Girona (2018b); Associació Guies de \\
Girona (2018); Vivó (1989)
\end{tabular}




\begin{tabular}{|l|l|}
\hline Título de la leyenda & Referencias dónde se menciona \\
\hline La cruz de fuego & Vivó (1989) \\
\hline $\begin{array}{l}\text { La Fuente de pericot (Las fuentes } \\
\text { prodigiosas) }\end{array}$ & Garcia (1956); Vivó (1989) \\
\hline La Leona & $\begin{array}{l}\text { Ajuntament de Girona (2018a); Ajuntament de } \\
\text { Girona (2018); Associació Guies de Girona (2018); } \\
\text { Garcia (1956) }\end{array}$ \\
\hline El ama de llaves de San Narciso & $\begin{array}{l}\text { Ajuntament de Girona (2018b); Associació Guies } \\
\text { de Girona (2018); Garcia y Cornellà (1956); Vivó } \\
\text { (1989) }\end{array}$ \\
\hline Las moscas de San Narciso & $\begin{array}{l}\text { Ajuntament de Girona (2018a); Associació Guies de } \\
\text { Girona (2018); Vivó (1989) }\end{array}$ \\
\hline San Félix y el Ladrón & Garcia y Cornellà (1956) \\
\hline
\end{tabular}

Fuente: Elaboración propia

La metodología escogida para hacer el análisis es de carácter cualitativo y se ha realizado siguiendo dos fases. En la primera fase, para el análisis de las leyendas, se ha utilizado la técnica del análisis de contenido, un método en auge dentro de las investigaciones de carácter social (Hall y Valentin, 2005). Este método se define como una técnica de investigación observacional que puede ser aplicada para analizar diferentes formas de contenido e identificar pautas, temáticas y significados dentro de un mismo elemento de comunicación (Camprubí y Coromina, 2016). Así pues, el análisis de contenido se puede considerar como una investigación observacional, que, utilizada de forma sistemática permite identificar de una forma simbólica cualquier tipo de contenido en un registro comunicativo. Este tipo de exploración ha sido utilizado también en el ámbito turístico (Hall y Valentin, 2005), permitiendo así procesar la información y transformar el contenido del elemento comunicativo objeto de estudio en datos resumidos y comparables, partiendo de una serie de reglas objetivas y de categorización de la información (Kassarjian, 1977). El análisis de contenido de las leyendas tiene como propósito poder caracterizar los elementos que se vinculan a la ciudad de Girona y que pueden aportar valor en la transmisión de la imagen de marca del destino y, asimismo, comprobar posteriormente si se están utilizando en la estrategia de comunicación y branding llevada a cabo desde los entes de promoción del destino.

Para llevar a cabo la categorización de las leyendas se han tomado como referencia las variables que Fog et al. (2010) y Yavuz et al. (2016) consideran fundamentales para elaborar un relato. Según estos autores cada historia se compone de elementos que pueden ser clasificados en distintos grupos y que se complementan entre ellos a la hora de elaborar un relato. Siguiendo estos autores se han considerado cinco categorías distintas (mensaje, conflicto, personajes, lugar y objetos) quedando sintetizadas en el cuadro 2. 


\section{Cuadro 2 \\ VARIABLES DE ANÁLISIS}

\begin{tabular}{|l|l|l|}
\hline Categorías & Descripción & Autores \\
\hline Mensaje & $\begin{array}{l}\text { El motivo por el cual la historia es } \\
\text { explicada, normalment una afirmación } \\
\text { moral que se ve reflejada a lo largo del } \\
\text { relato. }\end{array}$ & Fog et al. (2010) \\
\hline Conflicto & $\begin{array}{l}\text { La acción principal que transcurre a lo } \\
\text { largo de la historia, desde que se genera } \\
\text { un conflicto hasta que éste es resuelto. }\end{array}$ & Fog et al. (2010); Yavuz et al. (2016) \\
\hline Personajes & $\begin{array}{l}\text { Las diferentes figuras que aparecen en la } \\
\text { narración. }\end{array}$ & Fog et al. (2010); Yavuz et al. (2016) \\
\hline Lugar & $\begin{array}{l}\text { El espacio físico donde transcurre la } \\
\text { acción. }\end{array}$ & Yavuz et al. (2016) \\
\hline Objetos & $\begin{array}{l}\text { En este caso corresponden con los } \\
\text { atractivos turísticos de la ciudad que } \\
\text { aparecen mencionados en las leyendas }\end{array}$ & Yavuz et al. (2016) \\
\hline
\end{tabular}

Fuente: Elaboración propia.

Durante la categorización de los textos se han añadido subcategorías en las categorías “personajes" y "lugar". Para la categoría "personajes" se ha diferenciado entre personajes principales y secundarios; y para la variable lugar se ha diferenciado entre Girona y los alrededores de Girona, donde se incluyen las distintas poblaciones alrededor de la ciudad. Asimismo, también se ha creado una subcategoría para los distintos barrios de la ciudad. Esta subcategorización ha permitido precisar en el análisis y concretar la información identificada, para poder localizar de forma más precisa los espacios mencionados en los relatos.

Para llevar a cabo el análisis de contenido de los relatos se ha utilizado el programa Nvivo 11. Se trata de un programa utilizado de forma recurrente en las investigaciones de análisis de contenido, y que permite examinar los textos, codificar las palabras clave definidas para la investigación e identificarlas en los textos. El método ha sido aplicado de acuerdo lo establecido por Camprubí y Coromina (2016) para poder garantizar la objetividad, fiabilidad y reproducibilidad de esta investigación. El proceso de análisis ha estado realizado por parte de los dos investigadores que suscriben este artículo. Cuando se ha producido algún desacuerdo en la codificación se ha discutido el resultado hasta llegar a un acuerdo.

Así mismo, en la segunda fase se han llevado a cabo entrevistas semi-estructuradas con el fin de recabar información relativa a la estrategia de comunicación y branding de la ciudad de Girona, y en particular sobre el rol de las leyendas en el storytelling de la ciudad. Concretamente se han realizado entrevistas a un técnico del área de turismo del Ayuntamiento de Girona como ente de promoción local (LMO), a un técnico de la Oficina de Turismo de la ciudad y la persona responsable en la Asociación de Guías de Girona. El 
análisis de las entrevistas ha permitido comprender en qué situación se encuentra el uso de las leyendas de Girona en el contexto de la estrategia de marca de la ciudad.

\section{RESULTADOS}

En este apartado se presentan los resultados que se desprenden del análisis de las leyendas y las entrevistas realizadas a agentes claves de la ciudad de Girona. En el primer apartado, se presenta los resultados relativos del análisis de las leyendas; en el segundo apartado, se exponen los elementos más relevantes relacionados con la marca y el branding de la ciudad de Girona en los últimos años; y finalmente, en el último apartado, se destacan los resultados relativos al uso y/o potencialidades de las leyendas como parte del branding de la ciudad.

\subsection{Elementos clave en las Leyendas de Girona}

Derivado de la literatura académica y de la metodología expuesta en el apartado anterior, este apartado presenta los resultados relativos al análisis de las leyendas del caso de estudio seleccionado, considerando cinco variables distintas: (a) personajes, (b) lugar, (c) objeto, (d) conflicto o experiencia, y (e) mensaje.

\subsubsection{Personajes}

Los personajes son uno de los principales elementos de un relato, ya que permiten poder personificar la historia en un individuo real o ficticio, y que ayudará a la audiencia a empatizar, es decir, sentir emociones, y posiblemente sentirse identificados. A partir de los resultados del análisis de contenido (cuadro 3) podemos observar que la variable "personajes" se encuentra en todas las leyendas analizadas. Cada una de estas leyendas menciona unos personajes principales que se ven involucrados en la acción que transcurre durante el relato. Muchas de ellas también incluyen varios personajes secundarios que también participan en algún momento (62\%). Entre estos personajes se encuentra un gran número de personajes históricos o religiosos, junto con personajes inventados y animales de carácter mitológico. Uno de los personajes más recurrentes es "Carlomagno" que aparece en el 19\% de las leyendas. Otros personajes recurrentes de carácter religioso son San Narciso y la Virgen María; y en repetidas ocasiones los ciudadanos de la ciudad de Girona también toman su rol como personajes en los relatos. Según Salomón (2007), los personajes en el storytelling de marcas se convierten en un elemento clave para favorecer la comparación entre iguales (el personaje con su audiencia). Si bien es cierto que en este caso se trata de personajes históricos (p.ej. Carlomagno) o bien mitológicos (p.ej. La Cocollona), y es más difícil esta comparación entre iguales, sí que los personajes pueden despertar emociones y simpatías entre la audiencia. 


\subsubsection{Lugar}

La variable "lugar" está presente en un 95\% de las leyendas analizadas. Solo la leyenda de "La silla de Carlomagno" no identifica una localización concreta, en cambio todas las demás se sitúan en algún espacio específico en la ciudad o en poblaciones de su entorno. Principalmente, los lugares que se mencionan se encuentran en el centro histórico de la ciudad, es decir, calles o espacios más amplios. La concentración geográfica de los relatos puede verse como un elemento positivo que facilita la relación del espacio físico con el relato.

\subsubsection{Objeto}

Por otro lado, el número de "objetos" y atractivos turísticos que se mencionan en las leyendas también es elevado $(81 \%)$. En particular, sólo se han identificado 3 leyendas en las cuales no se especifica ningún elemento concreto. Estos puntos de interés turístico, igual como los espacios mencionados en las leyendas, también se encuentran mayoritariamente en el centro histórico de la ciudad, siendo muy recurrente la Catedral en casi la mitad de las leyendas (43\%). Este también es un factor muy importante ya que permite la materialización del relato. Desde el momento que hay un monumento o un hito que representan físicamente parte de la leyenda, esto favorece la conexión entre dicho elemento y su audiencia.

Con el fin de poder definir mejor la situación espacial de los distintos "lugares" y "objetos" mencionados, la figura 1 los sitúa en un plano de la ciudad, en el cuál el círculo representa el "lugar" mencionado en la leyenda y la estrella el "objeto" al qué se refiere. Se puede constatar una fuerte densidad, tanto de los "objetos" como "lugares", en el centro histórico de la ciudad. 
Figura 1

REPRESENTACIÓN DE LAS VARIABLES “LUGAR” Y “OBJETO” SOBRE EL PLANO DE LA CIUDAD DE GIRONA

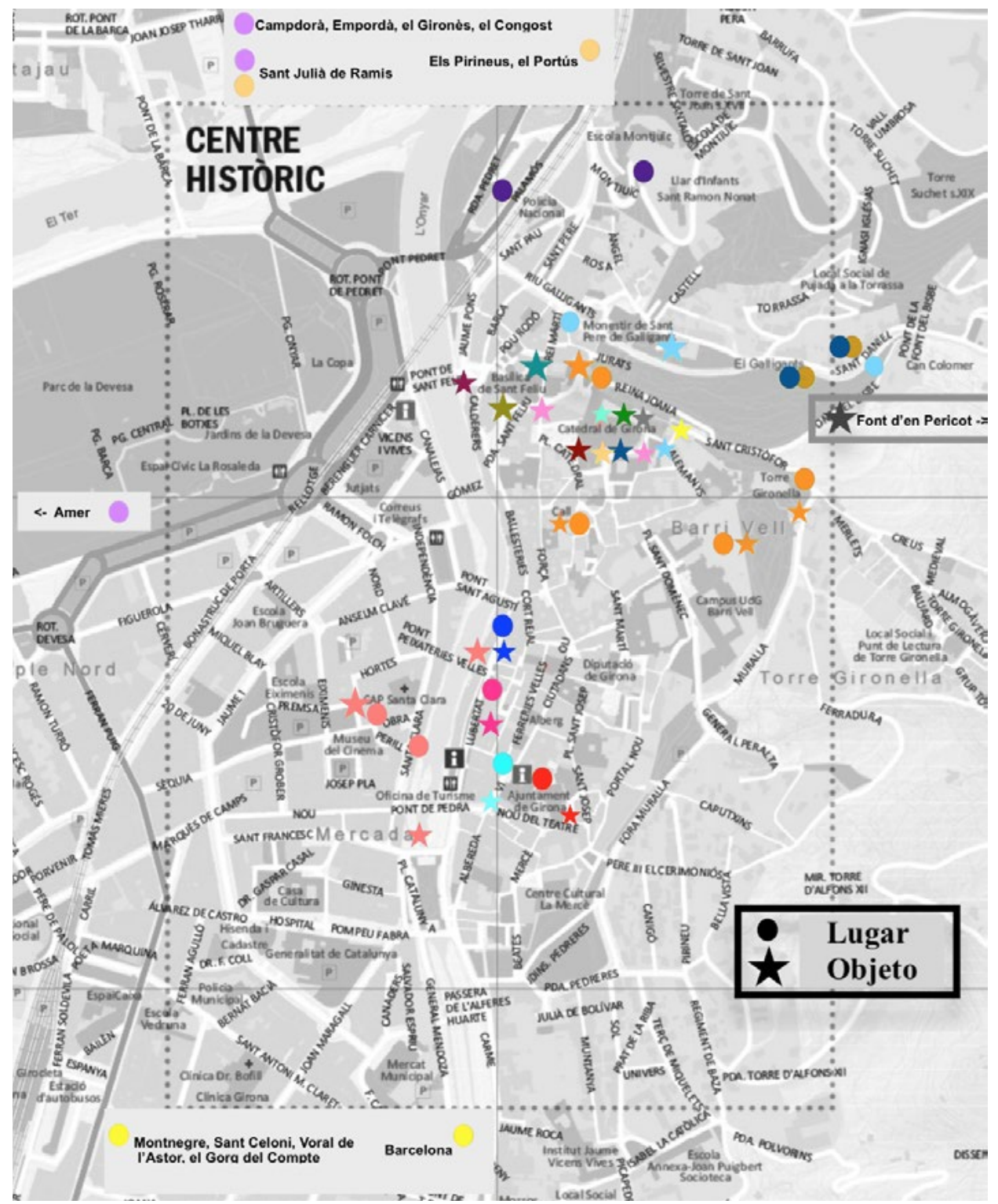

Fuente: Elaboración propia. 


\subsubsection{Conflicto o experiencia}

Tal como establecía Yavuz et al. (2016) en su estudio sobre la ciudad de Adana, el "conflicto o experiencia" es una variable clave en el desarrollo del storytelling, de modo que está presente de una forma u otra en cualquier relato. En los resultados de este estudio se observa que mayoritariamente aparecen conflictos de carácter histórico o religioso a través de los cuales se puede observar la gran vinculación de la población con la religión cristiana, ya que gran parte de los relatos muestran conflictos entre grupos de diferentes religiones o entre diferentes colectivos. Sólo la leyenda de "La Leona" no refleja ningún conflicto como tal en su narración, sino que cuenta una anécdota relacionada con las costumbres populares de Girona. Así pues, la leyenda explica que cualquier visitante de la ciudad de Girona que bese el culo de la leona (una antigua estatua que se encuentra a los pies de la iglesia de San Félix), volverá a la ciudad, dándole, por tanto, una vertiente más experiencial.

\subsubsection{Mensaje}

Finalmente, la variable "mensaje" corresponde con un valor del $48 \%$ ya que el contenido de las leyendas no solo incluyen afirmaciones ideológicas o morales, tal y como determinaba Fog et al. (2010), sino que en este caso también reflejan hechos históricos, tradiciones culturales y costumbres populares propios de la ciudad. Muchas de las leyendas hablan de hechos históricos y conflictos pasados sin llegar a transmitir un mensaje ético. Muchos de ellos son anécdotas y tradiciones populares conocidas por gran parte de la población gerundense.

\section{Cuadro 3}

\section{PRESENCIA DE LAS VARIABLES DE ANÁLISIS EN VALORES ABSOLUTOS Y PORCENTUALES}

\begin{tabular}{|l|l|l|}
\hline Variable & Frecuencia & Porcentaje \\
\hline Personajes & 21 & $100 \%$ \\
\hline Lugar & 20 & $95 \%$ \\
\hline Objeto & 17 & $81 \%$ \\
\hline Conflicto o experiencia & 21 & $100 \%$ \\
\hline Mensaje & 10 & $48 \%$ \\
\hline Total & 21 & $100 \%$ \\
\hline
\end{tabular}

Fuente: Elaboración propia. 
A partir de estos resultados obtenidos podemos afirmar que el contenido de los relatos analizados está compuesto por los elementos considerados esenciales en el storytelling según Fog et al. (2010) y Yavuz et al. (2016). Los resultados presentados muestran importantes similitudes con el estudio de la ciudad de Adana (Turquía) realizado por Yavuz et al. (2016), en el que destacan numerosos personajes, lugares y conflictos/experiencias. La gran diferencia se encuentra en la identificación de objetos específicos, los cuales son más comunes en el caso expuesto por Yavuz et al. (2016).

Paralelamente, podemos sostener que la elevada presencia de los elementos considerados esenciales (Mossberg et al., 2011; Frog et al., 2010; Yavuz et al., 2016), encontrándose en un porcentaje de más del $80 \%$ en la mayoría de los casos, confirma que las leyendas de Girona tienen la capacidad e ingredientes fundamentales para ser utilizados como herramienta de storytelling y como relatos aptos para ser el eje central de una estrategia de branding para la ciudad de Girona. Además, los lugares y objetos se sitúan en su gran mayoría en el centro histórico de la ciudad y se encuentran en una localización próxima a los atractivos turísticos más destacados, por lo que podrían perfectamente encajar de manera complementaria en una futura estrategia turística de la ciudad basada en el storytelling de dichas leyendas

\subsection{La marca y el branding de la ciudad de Girona}

A partir de las entrevistas realizadas a los distintos agentes clave del destino, se pudo obtener información relevante en relación a la política de promoción de la ciudad objeto de estudio. De las entrevistas se ha desprendido un creciente interés por mejorar los aspectos relacionados con la marca Girona, es decir, se destaca la necesidad de establecer una estrategia de marca que permita poder posicionar-se adecuadamente en sus principales mercados turísticos. No obstante, se pone de manifiesto la existencia de cierta dependencia a las decisiones políticas y los partidos políticos gobernantes: "Nuestra estrategia siempre va ligada con el partido político del momento, que es quien marca como quiere la ciudad en los próximos cuatro años" (E.1). De hecho, esto es uno de los elementos que comúnmente afectan a los destinos turísticos y añaden complejidad a la planificación estratégica y, más particularmente, al branding tal y como establece (Pike, 2005).

Desde el ente de promoción del turismo local (LMO) se señala el cambio político que tuvo lugar en 2004 como un punto de inflexión en el que se vislumbraban cambios en la dinámica de gestionar el turismo y, más particularmente, en la promoción turística de la ciudad. Fue en 2007 cuando se creó un área de turismo, es decir la actual LMO, tomando una actitud más "proactiva" tal y como explicaba en la entrevista: "En ese momento empezaron a ser un poco más proactivos, no sólo recibir a quien venía o lo pedía, sino que se empezó a ir a buscar el turista, se empezó a participar más en ferias, etc.” (E.1). En aquél entonces fue cuando se empezó a hablar de la necesidad de elaborar un Plan Estratégico turístico para que pusiera las bases del desarrollo y planificación turística de la ciudad, incluyendo por tanto los aspectos relacionados con el branding y la promoción. Años después, en 2018, se ha materializado la elaboración de este Plan 
Estratégico que ha supuesto un avance muy importante para establecer el rumbo turístico de la ciudad. Si embargo, es pronto para ver los efectos de este plan estratégico que está aún en una temprana fase de implementación.

Focalizando sobre los aspectos relacionados con las acciones de promoción y comunicación turística, se remarca una orientación clara hacia la búsqueda de un posicionamiento de la ciudad de Girona en el mercado inglés, y muy especialmente en el mercado francés; destacando que para este último ya se vienen realizando innumerables acciones desde el año 2016 con el fin de conseguir una mayor notoriedad. También se desprende de las entrevistas que anteriormente hubo una orientación hacia el mercado español en la que se consiguieron notables resultados: "Hace unos años estaba centrada también en el mercado español y la verdad es que se logró una cierta notoriedad e incrementó bastante su presencia" (E.1).

En relación con las herramientas de comunicación se destaca el esfuerzo que se ha realizado para utilizar los distintos canales online (página web y redes sociales), sin dejar de lado los canales tradicionales, tal y como se desprende de las entrevistas. En cuanto a las actividades de relaciones públicas llevadas a cabo destacan la participación a workshops, por ejemplo, con agencias de viajes receptivas; su presencia en ferias genéricas y/o especializadas; y la participación en viajes de familiarización, presstrips o blogtrips que en muchos casos son conjuntos con otros entes de promoción. Cabe destacar que desde el LMO se remarca la importancia de poder colaborar con otros entes de promoción ya sean a nivel regional o nacional, con el fin de poder aprovechar sinergias. Esta no es una práctica reciente, sino que se puede hablar de una consolidación de este tipo de colaboraciones, que ya se desprendían de los resultados de la tesis doctoral de Camprubí (2009).

En cuanto a la marca Girona, el año 2011 se creó la marca "Girona Emociona” que según la diagnosis del Plan Estratégico de la ciudad (2018: 20) tenía como objetivo "iniciar políticas de promoción para situar la ciudad en el imaginario colectivo en el ámbito internacional, y generar así empleo y riqueza". Así mismo, esta marca confluye con la marca "Girona, ciudad de festivales" creada el 2014 y gestionada desde el área de Cultura del consistorio de la ciudad con el propósito de aglutinar la oferta de 16 festivales que se llevan a cabo a lo largo del año; y su certificación por parte de la Agencia Catalana del Turismo bajo las marcas de "Destino de Turismo Deportivo" (DTE) y de "Ciudades con carácter". Cabe añadir que, en el reciente Plan Estratégico de turismo de la ciudad, en su reto 5 (p. 225) titulado "Mejorar el posicionamiento de Girona como destino y el valor añadido ofrecido" se destaca como objetivos el de "generar una estrategia sobre el relato de Girona" y al mismo tiempo "posicionar, proyectar y reforzar la marca Girona". Es este sentido, y considerando la coexistencia de distintas marcas y las indicaciones del Plan Estratégico en su "reto 5" se deja entrever la falta de una verdadera estrategia de branding. Una estrategia que establezca los elementos asociados a la identidad y valores de la marca de la ciudad, y que marque una ruta hacia el relato mencionado en el mismo Plan Estratégico como factor clave; y en su caso, que establezca una arquitectura y portafolio adecuado de las marcas que coexisten, con el fin de evitar distorsiones y dificultades en el posicionamiento de la marca de la ciudad. 


\subsection{Las leyendas y el branding de la ciudad de Girona}

Focalizando en los resultados de las entrevistas en relación a las Leyendas de Girona se especificó que a partir del año 1993 y durante unos cinco años se realizaron visitas guiadas teatralizadas de las Leyendas de la ciudad, a iniciativa de 3 guías pertenecientes a la Asociación de Guías de la ciudad. Este producto turístico obtuvo una fuerte popularidad gracias a una puesta en escena singular y el obsequio de productos de marchantaje que fortalecían los símbolos y conceptos transmitidos en algunas de las leyendas. Por ejemplo, un punto de libro con una representación de la leona y un beso o un lápiz con las moscas de San Narciso pintadas. Estos elementos permitieron sentar en el imaginario colectivo algunos símbolos, y relacionarlos con la imagen de la ciudad. En las entrevistas se menciona específicamente que "la iniciativa de las visitas de las leyendas resultó ser un formato muy auténtico ya que, por ejemplo, la estatua de la Leona no tenía escaleras y, los visitantes, para poder darle un beso debían escalar por su columna. Además, también incluyeron "efectos especiales" para hacerlas más originales. En el caso de la leyenda del Fantasma de la mujer judía, se incluyeron elementos para ambientarla como por ejemplo un sonido que simulaba su canto o tiraban una cabeza desde la Torre Gironella para simular su muerte" (E.2)."

En las entrevistas se puso de manifiesto que con el fin de estas visitas guiadas se paralizó la explotación de las leyendas en forma de producto turístico, quedando relegadas a un mero recurso cultural de la ciudad. Las visitas guiadas actuales no presentan las leyendas como parte esencial de las mismas, sino que simplemente mencionan algunos elementos procedentes de las leyendas como pueden ser algunas de las curiosidades que albergan. En las entrevistas se especificó que "ni los recorridos ni los discursos que se siguen son fijos, siempre se adaptan al grupo y al tiempo disponible y, de esta forma, cada visita incluye unas leyendas u otras" (E.2.). Desde la Asociación de Guías se desprende su interés por no dejar de lado las leyendas a través de visitas temáticas bajo petición, pero que distan de las originales visitas teatralizadas. Tal y como se especificaba en las entrevistas "también se realizan visitas a demanda para grupos de niños o adultos y, siempre que estén interesados en las leyendas, se incluyen en el recorrido para que también puedan conocerlas" (E.2). Actualmente, algún museo, como el Museo de Historia de Girona, ofrece actividades educativas dirigidas a escuelas que tratan también algunas de las leyendas de la ciudad; y en ocasiones específicas se han ofrecido actividades específicas para contribuir a la divulgación de las leyendas de la ciudad como parte de su patrimonio inmaterial. Bajo el punto de vista de Mossberg, et al. (2011) los productos turísticos, tales como visitas guiadas forman parte de la escenificación del storytelling que contribuye positivamente a la transmisión de valores del destino. Por ejemplo, la habilitación de unas escaleras para poder besar el culo de la leona, o la existencia de suvenires basados en representaciones de los objetos y personajes de las leyendas forman parte de la materialización de este relato en la experiencia del visitante de la ciudad, según Mossberg et al. . (2011).

En relación específica a la promoción actual de las leyendas, se ha visto que está limitada a estar presentes en la página web oficial de turismo y, para ser precisos, se especifican 11 de las 34 leyendas existentes en el apartado titulado "Guía Turística para niños" y se encuentran traducidas en 3 idiomas (catalán, castellano e inglés). También 
se mencionan en la web oficial del Ayuntamiento de la ciudad en el apartado "Datos del municipio" dónde se incluyen únicamente 7 leyendas. Así pues, las leyendas en la actualidad no se están utilizando en el relato de la comunicación de la marca Girona, aunque se consideran un recurso del patrimonio intangible de la ciudad. Es decir, no existe una comunicación estratégica de las leyendas en forma de storytelling que permita realzar los valores e identidad de la marca de la ciudad. Adicionalmente, si bien las entrevistas mostraron la necesidad de generar un relato único para la construcción de una marca fuerte, en ningún caso se especificó la intencionalidad de usar las Leyendas como base de este relato. Por tanto, se concluye una falta de aprovechamiento de este potente recurso, el cual se postula como una fuente de ventaja competitiva (Chronis, 2005; Mossberg, 2007).

\section{CONCLUSIONES}

Considerando la importancia del storytelling como herramienta altamente efectiva para determinar un eje central de narración a la hora de crear una marca (Salomon, 2007), y poder desarrollar ventajas competitivas ante otros destinos competidores (Chronis, 2005; Mossberg, 2007; Kim y Youn, 2017), este estudio pretendía explorar las potencialidades y el uso del storytelling en un destino turístico en base a relatos que forman parte de su patrimonio cultural inmaterial. Para ello se ha utilizado el caso de estudio de las leyendas la ciudad de Girona (España).

Los resultados han permitido demostrar que las leyendas de Girona incluyen los elementos determinados como necesarios y esenciales en el storytelling (Frog et al., 2010; Yavuz et al. , 2016). Es decir, objetos y lugares que se encuentran cerca los unos de los otros, contribuyendo a la materialización del storytelling y favoreciendo la conexión entre el relato y el visitante (Mossberg et al., 2011; Yavuz et al., 2016), y personajes que contribuyen a la conexión emocional con la audiencia (Salomon, 2007). Por lo tanto, se ha podido comprobar que las Leyendas de Girona son relatos que pueden ser utilizados como parte angular del storytelling en la estrategia de marca de la ciudad, es decir, los valores e identidad de la marca pueden estar fundamentados con ideas y símbolos surgidos de las leyendas, considerando su relevancia como patrimonio cultural inmaterial de la ciudad y sus residentes.

El storytelling al entorno de las leyendas de Girona sigue aún latente. Si bien, ya no existen las visitas teatralizadas que tanto éxito tuvieron, sigue habiendo elementos que permiten rememorar o materializar la conexión entre alguna de las leyendas y la experiencia turística, como el caso de las escaleras para besar la Leona, algunas alusiones a las leyendas durante las visitas guiadas, o bien los suvenires. Estos elementos, según Mossberg et al. (2011), son importantes para poder materializar la experiencia del visitante, y deben formar parte de la ejecución correcta del storytelling, tal como constataron estos autores en su estudio sobre 5 casos en países nórdicos.

Las entrevistas han mostrado evidencias del hecho que el potencial de las leyendas no se está utilizando para la estrategia de marca de la ciudad, tal y como suceden en el caso de las Leyendas de Limfjorden en Dinamarca (Mossberg et al., 2011), diferenciándose del caso de "The shelfish journey" en Suecia que si se ha utilizado para el desarrollo de la marca territorial (Mossberg et al., 2011). Si bien los resultados han dejado patente que 
los entrevistados consideran importante el desarrollo de un relato para la creación de la marca del destino, tal y como propone el Plan Estratégicos de turismo de la ciudad, actualmente aún no se ha llevado a cabo, y en ningún caso se mostró la intencionalidad de usar las leyendas como parte esencial de este relato. En este contexto, cabe destacar que los stakeholders anclados al territorio son una parte esencial de la estrategia de branding (Camprubí, 2009) basada en el storytelling. Mossberg et al. (2011) dejan patente que la coordinación y colaboración entre los distintos agentes implicados en el desarrollo del storytelling son fundamentales para poder garantizar su éxito. Además, Yavuz et al. (2016) destaca que el LMO debe ser quien lidere este proceso. En el caso analizado se ha podido constatar esta falta de acuerdo y trabajo común para promover y realzar la potencialidad de las leyendas, reavivarlas como producto turístico que ponga en valor el patrimonio de la ciudad, y sirva como base de la estratégica de marca.

Este artículo contribuye en la literatura académica aportando conocimiento sobre la potencial aplicabilidad del storytelling en el branding de marcas territoriales, y pone de manifiesto la necesidad de continuar en el estudio empírico en investigaciones futuras. Este estudio abre la puerta a nuevas investigaciones futuras sobre casos en los que sí se esté utilizando su legado cultural en forma de leyendas o relatos para el desarrollo de marcas territoriales, en las que se pueda indagar sobre aspectos como los recursos que se utilizan para desarrollar el storytelling, la efectividad del storytelling en el branding del destino, el proceso de desarrollo de la estrategia de branding basada en el storytelling, así como el rol y relaciones establecidas entre los stakeholders implicados. También deberían centrarse en determinar cuáles son los elementos procedentes de las leyendas que han quedado impregnados en los valores e identidad de la marca construida; y al mismo tiempo cuál es el mensaje que se transmite a través de las herramientas de comunicación. En definitiva, poder constatar la efectividad del stroytelling de un destino basado en sus propias leyendas.

\section{REFERENCIAS}

AAKER, D. A. (1998): Brands: Managing Brand Equity Brand value. 13th edition. Sao Paulo, Elisevier.

AJUNTAMENT DE GIRONA. (2018a, Abril 2018): «Dades del municipi de Girona». Disponible en http://www2.girona.cat/ciutat_dadesgirona_llegendes

AJUNTAMENT DE GIRONA. (2018b, Abril 2018): «Guía turística de Girona per a infants». Disponible en http://www.girona.cat/turisme_infants/cat/index.php?idcat=867

ANHOLD, S. (2010): «Definitions of place branding-Working towards a resolution», Place Branding and Public Diplomacy, vol. 6, pp. 1-10.

ASSOCIACIÓ GUIES DE GIRONA. (2018, Abril 2018): «Llegendes de Girona». Disponible en https://guiesdegirona.com/visites/llegendes-de-girona/

BALMER, J.M.T. (2001): «Corporate identity, corporate branding and corporate marketing - Seeing through the fog», European Journal of Marketing, vol. 35 (3-4), pp. 248-291. 
BALMER, J.M.T. (2010): «Explicating corporate brands and their management: Reflections and directions from 1995», Journal of Brand Management, vol. 18 (3), pp. 180-196.

BASTOS, W. y LEVY, S.J. (2012): «A history of the concept of branding: practice and theory», Journal of Historical Research in Marketing, vol. 4 (3), pp. 347-368.

BERGER, P.L. y LUCKMANN, T. (1966): The social construction of reality: A treatise in the sociology of knowledge. London, Penguin Books.

BLOMBÄCK, A. y RAMÍREZ-PASILLAS, M. (2012): «Exploring the logics of corporate brand identity formation», Corporate Communications: An International Journal, vol. 17 (1), pp. 7-28.

CAMPRUBÍ, R. (2009): La formació de la imatge turística induïda: el paper de les xarxes relacionals. Tesis doctoral. Disponible en http://hdl.handle.net/10803/7959.

CAMPRUBÍ, R. y COROMINA, L. (2016): «Content analysis in tourism research», Tourism Management Perspectives, vol. 18, pp. 134-140.

CAMPRUBI, R., GUIA, J. y COMAS, J. (2008): «Destination networks and induced tourism image», Tourism Review, vol. 63 (2), pp. 47-58.

CHAVES, E.D.P.S. (2017): «Identity, positioning, brand image and brand equity comparison: a vision about quality in brand management», Independent Journal of Management y Production, vol. 8 (4), pp.1.246-1.263.

CHIU, H.C. HSIEH, Y.C. y KUO, Y.C. (2012): «How to align your brand stories with your products», Journal of Retailing, vol. 88 (2), pp. 262-275.

CHRONIS, A. (2005): «Coconstructing Heritage at the Gettysburg Storyscape», Annals of Tourism Research, vol. 32 (2), pp. 386-406.

CHRONIS, A. (2008): «Co-constructing the Narrative Experience: Staging and Consuming the American Civil War at Gettysburg», Journal of Marketing Management, vol. 24 (122), pp. 5-27.

DE SAN EUGENIO, J. (2013): «Fundamentos conceptuales y teóricos para marcas de territorio». Boletín de la Asociación de Geógrafos Españoles, n ${ }^{\circ}$ 62, pp. 189-211.

FOG, K., BUDTZ, C. y YAKABOYLU, B. (2005): Storytelling. Branding in practice. Berlin, Heiddelberg: Springer.

FOG, K., BUDTZ, C., MUNCH, P. y BLANCHETTE, S. (2010): Storytelling. Branding in practice. Berlin, Heidelberg: Springer.

GARCIA, D. (1956): Llegendes de Girona. Itinerari pel Barri Vell. Girona, Ajuntament de Girona.

GOVERS, R., y GO, F.M. (2004): «Cultural Identities Constructed, Imagined and Experienced: A 3-gap Tourism Destination Image Model», Tourism, vol. 52 (2), pp. 165-182.

GOVERS, R. y GO, F.M. (2016): Place branding: Glocal, virtual and physical identities, constructed, imagined and experiences. Springer.

GRAVILI, S., ROSATO, P. e IAZZI, A. (2017): «Managing DMOs through Storytelling: A Model Proposal for Network and Value Co-creation in Tourism».,International Business Research, vol. 10 (7), pp. 8-24.

GUBER, P. (2007): «The Four Truths of the Storyteller», Harvard Business Review, vol. 85 (12), p. 52. 
HALL, C.M. y VALENTIN, A. (2005): «Content Analysis», en Tourism Research Methods. Cabi, pp. 191-209.

KASSARJIAN, H.H. (1977): «Content Analysis in Consumer Research», Journal of Consumer Research, vol. 4 (1), pp. 8-18.

KAVARATZIS, M. y HATCH, M.J. (2013): «The dynamics of place brands: An identitybased approach to place branding theory», Marketing theory, vol. 13 (1), pp. 69-86.

KENSINGER, E.A. y CORKIN, S. (2003): «Effect of negative emotional content on working memory and long-term memory», Emotion, vol. 3 (4), pp. 378-393.

KESKIN, H., AKGUN, A. E., ZEHIR, C., AYAR, H. y KESKIN, H. (2016): «Tales of Cities: City Branding Through Storytelling», Journal of Global Strategic Management, vol. 1 (1), pp. 31-41.

KIM, J. H. y YOUN, H. (2017): «How to Design and Deliver Stories about Tourism Destinations», Journal of Travel Research, vol. 56 (6), pp.808-820.

KOTLER, P. (2011): Marketing Management: Analysis, planning, implementation and control. 5th ed. Sao Paulo, Atlas.

KOTLER, P., HAIDER, D.H. y REIN, I. (1993): Marketing Places: Attracting Investment. Industry, and Tourism to Cities, States, and Nations. New York, The Free Press.

MELER, M. y RUZIC, D. (1999): «Marketing identity of the tourist product of the Republic of Croatia», Tourism Management, vol. 20 (5), pp. 635-643.

MILIČEVIĆ, K., MIHALIČ, T. y SEVER, I. (2017), «An investigation of the relationship between destination branding and destination competitiveness», Journal of Travel y Tourism Marketing, vol. 34 (2), pp. 209-221.

MOSSBERG, L., THERKELSEN, A., H HUIJBENS, E. y BJÖRK AND OLSSON, A.K. (2011); Storytelling and destination development. Disponible en http://www.divaportal.org/smash/get/diva2:707209/FULLTEXT01.pdf

PARK, C.W., JAWORSKI, B.J. y MACINNIS, D.J. (1986): «Strategic Brand ConceptImage Management», Journal of Marketing, vol. 50 (4), pp. 135-145.

PIKE, S. (2005): «Tourism destination branding complexity», Journal of Product y Brand Management, vol. 14 (4), pp. 258-259.

PÎNZARU, F. (2012): «From conjunctural urban storytelling to city branding: An empirical model for bucharest», Management Research and Practice, vol. 4 (2), pp. 33-44.

PULIZZI, J. (2012): «The rise of storytelling as the new marketing», Publishing Research Quarterly, vol. 28 (2), pp. 116-123.

QU, H., KIM, L.H. e IM, H.H. (2011): «A model of destination branding: Integrating the concepts of the branding and destination image», Tourism Management, vol. 32 (3), pp. 465-476.

RAMZY,A. (2007): «The Leader's Guide to Storytelling. Mastering the Art and Discipline of Business Narrative», Corporate Reputation Review, vol. 10 (2), pp. 154-157.

ROONEY, J.A. (1995): «Branding: a trend for today and tomorrow», Journal of Product y Brand Management, vol. 4 (4), pp. 48-55.

RUIZ, E. y HERNÁNDEZ, M. (2007): «Identity and community - Reflections on the development of mining heritage tourism in Southern Spain», Tourism Management, vol. 28, pp. 677-687. 
SALMON, C. (2007): Storytelling: la machine à fabriquer des histoires et à formater les esprits. Paris, Éditions La Découverte.

URDE, M. (2013). «The corporate brand identity matrix», Journal of Brand Management, vol. 20 (9), pp. 742-761.

URRY, J. (1995). Consuming places. Routledge.

VAN REKOM, J. y GO, F. (2006). «Being discovered: A blessing to local identities?», Annals of Tourism Research, vol. 33 (3), pp. 767-784.

VELILLA, J. (2010). Branding tendencias y retos en la comunicación de marca. Barcelona, Editorial UOC.

VIVÓ, C. (1989). Llegendes i misteris de Girona. Quaderns de la Revista de Girona. Girona, Diputació de Girona / Caixa de Girona

WOODSIDE, A.G. y MEGEHEE, C.M. (2010). «Advancing consumer behaviour theory in tourism via visual narrative art. International», Journal of Tourism Research, vol. $12(5)$, pp. 418-431.

YAVUZ, M.C. SUMBUL, M., ELPEZE, N. y DERDIYOK, C.I. (2016). «Storytelling in destination brand communication: A qualitative analysis», Journal of Global Business Insights, vol. 1 (2), pp. 63-72. 
